\title{
Evaluation of the efficacy of maintenance therapy for low-to-intermediate-risk acute promyelocytic leukemia in molecular remission: A retrospective single-institution study
}

\author{
MASAHIDE YAMAMOTO, KEIGO OKADA, HIROKI AKIYAMA, TETSUYA KUROSU and OSAMU MIURA \\ Department of Hematology, Graduate School of Medical and Dental Sciences, \\ Tokyo Medical and Dental University, Tokyo 113-8519, Japan
}

Received October 20, 2014; Accepted November 28, 2014

DOI: $10.3892 /$ mco.2014.476

\begin{abstract}
The prognosis of acute promyelocytic leukemia (APL) has become the most favorable among all acute myeloid leukemias, due to the efficacy of treatment with all-trans retinoic acid (ATRA). ATRA combined with anthracycline-based chemotherapy has significantly improved the long-term outcome for low-to-intermediate-risk APL patients; thus, the efficacy of maintenance therapy for patients achieving molecular complete remission (MCR) following consolidation therapy has become debatable. To evaluate the efficacy of maintenance therapy, we conducted a retrospective analysis of 11 consecutive patients with low-to-intermediate-risk APL who received induction and consolidation therapy with ATRA and anthracyclines according to the PETHEMA LPA protocols at our hospital between January, 2001 and March, 2013. All the patients achieved MCR following consolidation therapy. Of these patients, 7 were followed without maintenance therapy, including 2 patients who discontinued maintenance therapy within 2 months. With a median follow-up of 85 months, the overall survival for all the patients was $100 \%$, while the disease-free survival estimate at 5 years with and without maintenance therapy was 100 and $85.7 \%$, respectively; the difference was not statistically significant $(\mathrm{P}=0.45)$. Two patients treated with maintenance therapy later developed secondary primary malignancy. Thus, even without maintenance therapy, ATRA combined with anthracyclines exhibited significant efficacy in low-to-intermediate-risk APL patients, suggesting that maintenance therapy, which is associated with adverse events, may be dispensable for patients achieving MCR following adequate consolidation therapy.
\end{abstract}

Correspondence to: Professor Osamu Miura, Department of Hematology, Graduate School of Medical and Dental Sciences, Tokyo Medical and Dental University, 1-5-45 Yushima, Bunkyo-ku, Tokyo 113-8519, Japan

E-mail: miura.hema@tmd.ac.jp

Key words: acute promyelocytic leukemia, maintenance therapy, all-trans retinoic acid, molecular remission, anthracycline

\section{Introduction}

Acute promyelocytic leukemia (APL) is a subtype of acute myeloid leukemia (AML) characterized by the presence of the $P M L / R A R A$ fusion gene, which is formed by the reciprocal chromosomal translocation $\mathrm{t}(15 ; 17)(\mathrm{q} 22 ; \mathrm{q} 21)$ (1). The prognosis of APL was previously the worst among all the types of AML, mostly due to the almost inevitable occurrence of disseminated intravascular coagulation at diagnosis or during chemotherapy. However, all-trans retinoic acid (ATRA) has significantly improved the prognosis of APL, which has now become a highly curable disease with ATRA and anthracycline-based chemotherapy (1). This combination results in complete remission (CR) rates of $\leq 95 \%$, with $>80 \%$ of the patients surviving without relapse. Thus, the combination of ATRA and chemotherapy is currently considered to be the standard treatment for newly diagnosed APL (1).

One of the major concerns regarding the treatment of low-to-intermediate-risk APL are currently treatment-related adverse events, which led to reduction of the anthracycline dose during consolidation therapy in the PETHEMA LPA2005 protocol compared to the LPA96/99, without any compromise in disease-free survival (DFS) or overall survival (OS) (2). Adverse events are also expected to occur during maintenance therapy, which is included in a number of clinical trials and continues for $\sim 2$ years. Of note, DFS was similar between the maintenance and non-maintenance groups in patients who achieved molecular CR (MCR) following consolidation therapy in the AIDA0493 trial (3). Very recently, a new combination therapy consisting of ATRA and arsenic trioxide (ATO) was associated with fewer treatment-related toxicities, with possibly better therapeutic effects compared to ATRA plus chemotherapy (4). Furthermore, for low-to-intermediate-risk patients, ATRA plus ATO without maintenance therapy was found to be at least comparable to ATRA plus chemotherapy with maintenance therapy (4). Therefore, maintenance therapy may not be essential for low-to-intermediate-risk patients who have achieved MCR following efficient induction and consolidation therapy.

To evaluate the clinical efficacy of maintenance therapy for such patients, we retrospectively analyzed the clinical outcomes of 11 consecutive low-to-intermediate-risk APL 
patients in MCR, with and without subsequent maintenance therapy.

\section{Patients and methods}

Patients. We retrospectively analyzed 11 consecutive cases of newly diagnosed low-to-intermediate-risk APL patients who received induction and consolidation therapy according to the PETHEMA LPA protocols $(5,6)$ between January, 2001 and March, 2013 at Tokyo Medical and Dental University Hospital, Tokyo, Japan. All the patients were morphologically diagnosed with APL and confirmed by reverse transcription-polymerase chain reaction (RT-PCR) for the $P M L / R A R A$ rearrangement. According to the guideline published by the Ministry of Health, Labour and Welfare of Japan, this retrospective study was approved by the Institutional Review Board of the Tokyo Medical and Dental University.

Treatment protocol and risk classification. The details of the PETHEMA LPA protocol (LPA96, LPA99 and LPA2005) regimens have been previously published $(2,5)$. In brief, induction therapy consisted of ATRA with idarubicin (IDA). Patients who achieved CR received 3 monthly consolidation courses with ATRA and anthracycline-based chemotherapy. Maintenance therapy, consisting of mercaptopurine, methotrexate and ATRA, was continued for 2 years. According to the relapse risk categories used by the joint PETHEMA-GIMEMA study (6), low-to-intermediate risk was defined as a white blood cell count at diagnosis of $\leq 10,000 / \mu 1$.

Statistical analysis. MCR was defined as negativity for $P M L / R A R A$ by RT-PCR. Relapse was defined as the re-appearance of morphologically abnormal leukemic cells following initial clearance of the marrow or extramedullary sites. OS and event-free survival (EFS) were calculated from the date of induction therapy initiation, while DFS was calculate from the date of $\mathrm{CR}$ achievement. The event was defined as relapse, death, or secondary primary malignancy (SPM) for EFS and relapse or death for DFS. Time-to-event analyses were performed using the Kaplan-Meier estimate and comparisons were performed using log-rank tests. All the statistical analyses were performed with EZR (Saitama Medical Center, Jichi Medical University, Saitama, Japan), which is a graphical user interface for R (The R Foundation for Statistical Computing) (7). The patients who had received maintenance therapy for $<3$ months were classified into the maintenance therapy-free group.

\section{Results}

Patient characteristics and responses. A total of 12 patients were diagnosed with low-to-intermediate-risk APL between January, 2001 and March, 2013 in our hospital. One patient received only induction therapy due to severe infection and was excluded from the following analyses. The patient characteristics are summarized in Tables I and II. According to the LPA risk-classification (6), 3 patients were classified into the low-risk and 8 patients into the intermediate-risk group. There were 3 internal tandem duplications of FLT3-positive patients and no D835Y-positive patient. All the patients achieved CR
Table I. Patient characteristics $(n=11)$.

\begin{tabular}{lcc}
\hline & \multicolumn{2}{c}{ Maintenance therapy } \\
\cline { 2 - 3 } Variables & Yes & No \\
\hline Gender & & 3 \\
Male & 2 & 4 \\
Female & 2 & \\
Age, years & $26-71$ & $19-81$ \\
Range & 66 & 46 \\
Median & & \\
WBC count/ $\mu 1$ & $700-6,800$ & $600-4,200$ \\
Range & 1,200 & 1,500 \\
Median & & $0.4-11.7$ \\
PLT count x10 $/ \mu 1$ & $1.1-6.7$ & 2.5 \\
Range & 2.3 & 2 \\
Median & & 5 \\
Risk & 1 & 36 \\
Low & 3 & 110 \\
Intermediate & 40 & \\
Days to CR, median & 136 & \\
Days to MCR, median & & \\
\hline
\end{tabular}

WBC, white blood cell; PLT, platelet; CR, complete remission; MCR, molecular CR.

following induction therapy (Table II). The $P M L / R A R A$ transcript levels became undetectable by quantitative RT-PCR in all the patients following consolidation-2 (Table III). Furthermore, the nested RT-PCR method failed to detect the PML/RARA transcript in all 10 cases examined by this method (Table III).

Maintenance therapy. Although 6 patients were initiated on maintenance therapy, 2 patients discontinued the therapy within 2 months due to adverse events, myelosuppression and infection (Table III). These 2 patients were classified into the group not receiving maintenance therapy.

Relapse and survival. With a median follow-up of 85 months, all the patients remain alive and APL relapsed in only 1 patient who did not received maintenance therapy (Table III). In this patient, meningeal involvement by APL was suspected on brain magnetic resonance imaging (MRI) during consolidation-3 therapy. The bone marrow revealed molecular relapse within 2 months after completing consolidation-3 therapy (day 269), followed by central nervous system relapse confirmed by cerebrospinal fluid examination on day 388 . Following salvage chemotherapy, the patient received umbilical cord blood transplantation and remains alive without relapse. As shown in Fig. 1A, no significant difference in the 5-year estimate of DFS was observed between the maintenance and maintenance-free group (100 vs. $87.5 \%, \mathrm{P}=0.45)$.

$S P M$. SPM was diagnosed in 2 patients in the maintenance group (Table III). One patient with colon cancer was treated 
Table II. Clinical characteristics of 11 patients.

\begin{tabular}{|c|c|c|c|c|c|c|c|c|c|c|c|c|c|c|}
\hline \multirow[b]{2}{*}{$\begin{array}{l}\text { Case } \\
\text { no. }\end{array}$} & \multicolumn{2}{|c|}{ Patient profile } & \multicolumn{7}{|c|}{ Leukemia profile } & \multicolumn{5}{|c|}{ Induction therapy } \\
\hline & $\begin{array}{l}\text { Gender/ } \\
\text { age (yrs) }\end{array}$ & $\begin{array}{c}\text { Past } \\
\text { history }\end{array}$ & $\begin{array}{l}\mathrm{WBC} \\
/ \mu 1 \mathrm{x}\end{array}$ & $\begin{array}{r}\text { PLT } \\
10^{4} / \mu 1\end{array}$ & Risk & ACA & CD34 & CD56 & $\begin{array}{l}\text { FLT3/ } \\
\text { ITD }\end{array}$ & Protocol & DIC & DS & $\begin{array}{c}\mathrm{CR} \\
\text { (days) }\end{array}$ & $\begin{array}{l}\text { Adverse } \\
\text { events }\end{array}$ \\
\hline \multicolumn{15}{|l|}{ MT } \\
\hline 1 & $\mathrm{M} / 63$ & & 700 & 1.1 & Int & + & - & - & - & LPA96 & + & + & 50 & $\mathrm{FN}$ \\
\hline 2 & $F / 26$ & & 6,800 & 1.2 & Int & NA & + & - & + & LPA96 & + & + & 39 & Sepsis \\
\hline 3 & $\mathrm{~F} / 69$ & Colon $\mathrm{Ca}$ & 1,300 & 3.4 & Int & - & NA & NA & - & LPA99 & + & - & 41 & $\begin{array}{l}\text { Genital } \\
\text { ulcer }\end{array}$ \\
\hline 4 & $\mathrm{M} / 71$ & & 1,100 & 6.7 & Low & + & NA & NA & NA & LPA99 & - & - & 36 & $\mathrm{FN}$ \\
\hline \multicolumn{15}{|c|}{ No MT } \\
\hline 5 & $\mathrm{M} / 19$ & & 600 & 4.2 & Low & NA & NA & NA & - & LPA96 & + & - & 58 & HPS \\
\hline 6 & $\mathrm{M} / 54$ & & 1,500 & 0.4 & Int & NA & NA & NA & NA & LPA96 & + & + & 34 & Infection \\
\hline 7 & $\mathrm{~F} / 32$ & & 4,200 & 2.5 & Int & + & + & - & - & LPA99 & + & + & 38 & \\
\hline 8 & $\mathrm{~F} / 81$ & $\begin{array}{c}\mathrm{RA} \\
\text { sarcoidosis }\end{array}$ & 1,300 & 11.7 & Low & + & + & - & NA & LPA99 & + & + & 36 & $\mathrm{FN}$ \\
\hline 9 & $\mathrm{M} / 62$ & Af & 1,800 & 2.5 & Int & + & - & - & + & LPA2005 & + & - & 34 & $\begin{array}{l}\text { Liver } \\
\text { dysfunction }\end{array}$ \\
\hline 10 & $\mathrm{~F} / 28$ & $\begin{array}{l}\text { Eating } \\
\text { disorder }\end{array}$ & 3,800 & 3.7 & Int & + & + & - & + & LPA2005 & + & - & 31 & $\begin{array}{l}\text { Pericoronitis } \\
\text { of wisdom } \\
\text { tooth }\end{array}$ \\
\hline 11 & $\mathrm{~F} / 46$ & Myoma & 1,100 & 0.4 & Int & - & - & - & - & LPA2005 & + & + & 45 & $\mathrm{FN}$ \\
\hline
\end{tabular}

MT, maintenance therapy; WBC, white blood cell; PLT, platelet; ACA, additional chromosomal abnormality; FLT3/ITD, internal tandem duplications of FLT3; DIC, disseminated intravascular coagulation; DS, differentiation syndrome; CR, complete remission; M, male; F, female; Int, intermediate; FN, febrile neutropenia; NA, not assessed; Ca, cancer; HPS, hemophagocytic syndrome; RA, rheumatoid arthritis; Af, atrial fibrillation.

Table III. Clinical course of 11 patients.

\begin{tabular}{|c|c|c|c|c|c|c|}
\hline \multirow[b]{2}{*}{ Case no. } & \multicolumn{3}{|c|}{$P M L / R A R A$ transcript level } & \multicolumn{3}{|c|}{ Long-term follow-up } \\
\hline & $\begin{array}{l}\text { RT-qPCR } \\
\text { negative }\end{array}$ & $\begin{array}{c}\text { Molecular } \\
\text { CR }\end{array}$ & $\begin{array}{l}\text { Molecular } \\
\text { relapse }\end{array}$ & Relapse & $\begin{array}{c}\text { SPM } \\
\text { (months) }\end{array}$ & $\begin{array}{c}\text { Follow-up } \\
\text { (months) }\end{array}$ \\
\hline \multicolumn{7}{|l|}{ MT } \\
\hline 1 & Ind & Ind & & & Colon Ca (69) & 137 \\
\hline 2 & Ind & $\mathrm{C}-2$ & & & & 126 \\
\hline 3 & $\mathrm{C}-1$ & $\mathrm{C}-2$ & & & Lung Ca (38) & 97 \\
\hline 4 & $\mathrm{C}-2$ & $\mathrm{C}-2$ & & & & 70 \\
\hline \multicolumn{7}{|l|}{ No MT } \\
\hline $5^{a}$ & Ind & Ind & & & & 102 \\
\hline $6^{\mathrm{b}}$ & $\mathrm{C}-1$ & $\mathrm{C}-2$ & & & & 85 \\
\hline $7^{\mathrm{c}}$ & Ind & $\mathrm{C}-2$ & Yes (day 269) & Yes (CNS, day 388) & & 95 \\
\hline 8 & C-1 & NA & & & & 27 \\
\hline 9 & Ind & C-1 & & & & 30 \\
\hline 10 & $\mathrm{C}-1$ & $\mathrm{C}-3$ & & & & 28 \\
\hline 11 & Ind & $\mathrm{C}-1$ & & & & 18 \\
\hline
\end{tabular}


A

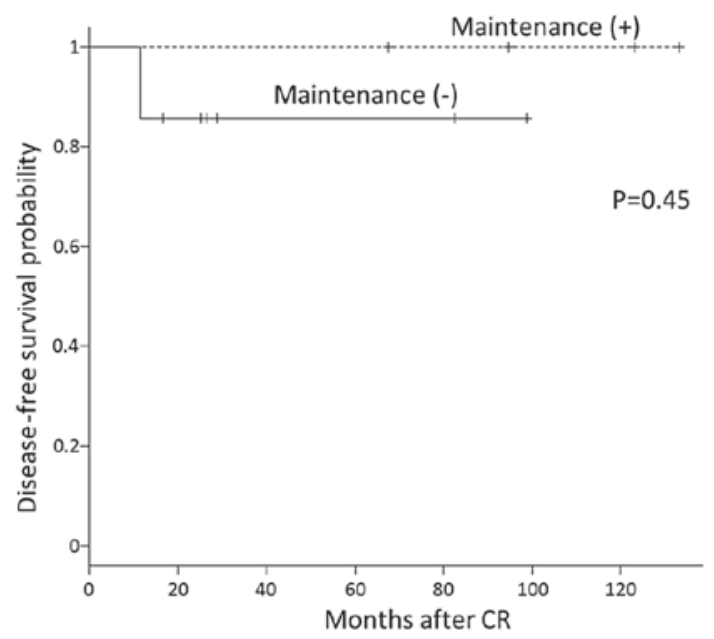

B

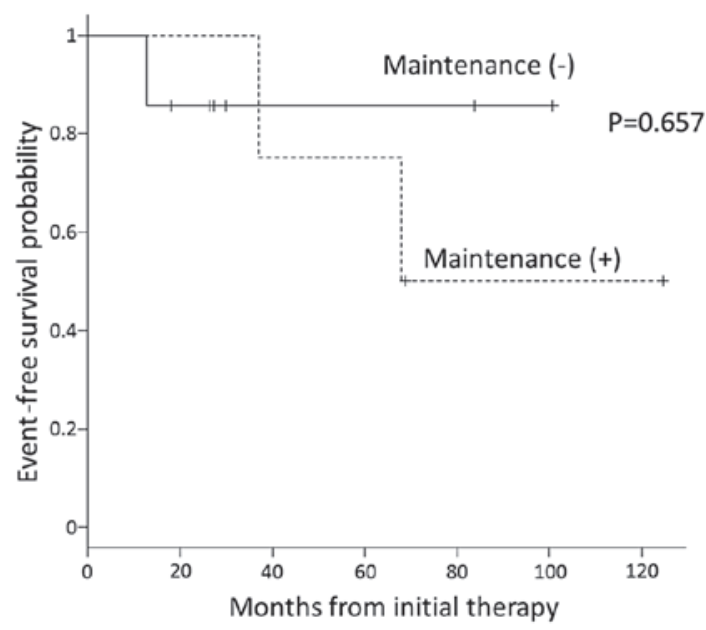

Figure 1. Kaplan-Meier estimates of (A) disease-free survival and (B) event-free survival for patients treated with and without maintenance therapy. CR, complete remission.

by curative colon resection and remains alive without relapse. In the other case, lung cancer was treated by lung resection but relapsed in the brain. Thus, the 5-year estimate of EFS for the maintenance and maintenance-free groups was 75 and $85.7 \%$, respectively; however, the difference was not statistically significant, as shown in Fig. 1B ( $\mathrm{P}=0.657)$.

\section{Discussion}

In the present study, we retrospectively evaluated the efficacy of maintenance therapy in low-to-intermediate-risk APL patients consecutively treated in our hospital according to the LPA protocols, who achieved molecular remission following consolidation therapy. The 5-year OS was $100 \%$ in both the maintenance and observation groups. Although 1 patient without maintenance therapy relapsed, the 5-year PFS for patients with and without maintenance therapy was 100 and $85.7 \%$, respectively, without a statistically significant difference $(\mathrm{P}=0.45)$. It should also be noted that, although the relapsed case exhibited MCR in the bone marrow sample following consolidation therapy, the MRI examination during the last course of consolidation had actually suggested meningeal relapse. APL relapsed in the bone marrow within 2 months after completing the consolidation therapy, followed by meningeal relapse confirmed by cerebrospinal fluid examination. Thus, albeit in MCR, this patient apparently had a significant amount of residual APL cells in sanctuary sites at the completition of consolidation therapy, suggesting that relapse could not have been prevented by low-dose maintenance therapy. Taken together, these clinical data strongly suggest that maintenance therapy may not be required to prevent relapse for low-to-intermediate-risk APL patients who were treated efficiently by ATRA and anthracycline-based therapy and achieved MCR.

In contrast to our data, 2 randomized studies previously reported the significant survival benefit of maintenance therapy with ATRA and/or low-dose chemotherapy $(8,9)$, which was later confirmed in the long-term outcome analyses $(10,11)$. However, in those studies, the patients were not treated concur- rently with ATRA and chemotherapy as induction therapy, or did not receive ATRA during consolidation therapy. Moreover, the patients were not examined for MCR or analyzed according to the current risk classification. Thus, it is hypothesized that maintenance therapy may not confer a survival benefit selectively in low-to-intermediate-risk APL patients who were treated with concurrent ATRA and anthracycline-based chemotherapy for induction and consolidation and achieved MCR. In accordance with this, a recent study reported that no advantage in terms of DFS was obtained by maintenance therapy in APL patients achieving MCR by the AIDA 0493 protocol, which includes the concurrent administration of ATRA and IDA as induction therapy, similar to the LPA protocols we employed (3). Furthermore, a recent controlled study demonstrated that none of the low-to-intermediate-risk APL patients treated with a consolidation regimen including ATO and achieving MCR relapsed, with or without maintenance therapy (12). Taken together, these results strongly suggest that maintenance therapy may not provide any benefit for low-to-intermediate-risk APL patients treated with efficient induction and consolidation regimens to achieve MCR.

Maintenance therapy did not provide any significant survival benefit; however, it was associated with adverse events in the present study. Maintenance therapy was initiated but abandoned within 2 months in 2 patients due to myelosuppression and infection (Table III). In this regard, fatal infections were observed in $2.5 \%$ of the patients randomized to the maintenance therapy group in a previous study investigating the efficacy of maintenance therapy (11). Another previous study on the AIDA 0493 protocol also reported sepsis and death due to infection or hemorrhage during maintenance therapy (3). Thus, the 2 patients were followed without further attempt at maintenance therapy and observed without relapse. It is noteworthy that, of the 4 patients receiving maintenance therapy, 2 patients later developed colon or lung cancer, while SPM was not reported in the observation group. Of note, in the Japan Adult Leukemia Study Group APL97 trial, intensified maintenance chemotherapy was shown to significantly compromise the OS of APL patients achieving MCR following 
consolidation therapy (13). In that trial, 2 patients in the maintenance group later developed therapy-related leukemia and succumbed to the disease. Furthermore, it was suggested that intensified maintenance therapy may have compromised the sensitivity of APL cells and the tolerance of patients to subsequent chemotherapy, thereby shortening survival following relapse (13). These results suggest that maintenance therapy may actually worsen the prognosis of low-to-intermediate-risk APL patients in MCR due to its toxicity.

In conclusion, the present study, conducted on a well-defined cohort of patients treated at a single institution, albeit being a retrospective analysis of a limited number of patients, strongly supports the emerging idea suggested by previous studies (11-13) that maintenance therapy may not be required or should be discouraged for low-to-intermediate-risk APL patients treated efficiently to achieve MCR, as it may be associated with adverse events, without exerting a significant preventive effect on leukemia relapse.

\section{References}

1. Elbahesh E, Patel N and Tabbara IA: Treatment of acute promyelocytic leukemia. Anticancer Res 34: 1507-1517, 2014.

2. Sanz MA, Montesinos P, Rayon C, et al; PETHEMA and HOVON Groups: Risk-adapted treatment of acute promyelocytic leukemia based on all-trans retinoic acid and anthracycline with addition of cytarabine in consolidation therapy for high-risk patients: further improvements in treatment outcome. Blood 115: 5137-5146, 2010.

3. Avvisati G, Lo-Coco F, Paoloni FP, et al; GIMEMA, AIEOP, and EORTC Cooperative Groups: AIDA 0493 protocol for newly diagnosed acute promyelocytic leukemia: very long-term results and role of maintenance. Blood 117: 4716-4725, 2011.

4. Lo-Coco F, Avvisati G, Vignetti M, et al; Gruppo Italiano Malattie Ematologiche dell'Adulto; German-Austrian Acute Myeloid Leukemia Study Group; Study Alliance Leukemia: Retinoic acid and arsenic trioxide for acute promyelocytic leukemia. N Engl J Med 369: 111-121, 2013.
5. Sanz MA, Martin G, Rayon C, et al: A modified AIDA protocol with anthracycline-based consolidation results in high antileukemic efficacy and reduced toxicity in newly diagnosed PML/RARalpha-positive acute promyelocytic leukemia. PETHEMA group. Blood 94: 3015-3021, 1999.

6. Sanz MA, Lo Coco F, Martin G, et al: Definition of relapse risk and role of nonanthracycline drugs for consolidation in patients with acute promyelocytic leukemia: a joint study of the PETHEMA and GIMEMA cooperative groups. Blood 96: 1247-1253, 2000.

7. Kanda Y: Investigation of the freely available easy-to-use software 'EZR' for medical statistics. Bone Marrow Transplant 48: 452-458, 2013.

8. Tallman MS, Andersen JW, Schiffer CA, et al: All-trans-retinoic acid in acute promyelocytic leukemia. N Engl J Med 337: 1021-1028, 1997.

9. Fenaux $\mathrm{P}$, Chastang $\mathrm{C}$, Chevret $\mathrm{S}$, et al: A randomized comparison of all transretinoic acid (ATRA) followed by chemotherapy and ATRA plus chemotherapy and the role of maintenance therapy in newly diagnosed acute promyelocytic leukemia. The European APL Group. Blood 94: 1192-1200, 1999.

10. Tallman MS, Andersen JW, Schiffer CA, et al: All-trans retinoic acid in acute promyelocytic leukemia: long-term outcome and prognostic factor analysis from the North American Intergroup protocol. Blood 100: 4298-4302, 2002.

11. Ades L, Guerci A, Raffoux E, et al; European APL Group: Very long-term outcome of acute promyelocytic leukemia after treatment with all-trans retinoic acid and chemotherapy: the European APL Group experience. Blood 115: 1690-1696, 2010.

12. Coutre SE, Othus M, Powell B, et al: Arsenic trioxide during consolidation for patients with previously untreated low/intermediate risk acute promyelocytic leukaemia may eliminate the need for maintenance therapy. Br J Haematol 165: 497-503, 2014.

13. Asou N, Kishimoto Y, Kiyoi H, et al; the Japan Adult Leukemia Study Group: A randomized study with or without intensified maintenance chemotherapy in patients with acute promyelocytic leukemia who have become negative for PML-RARalpha transcript after consolidation therapy: the Japan Adult Leukemia Study Group (JALSG) APL97 study. Blood 110: 59-66, 2007. 